\begin{tabular}{|c|c|c|}
\hline 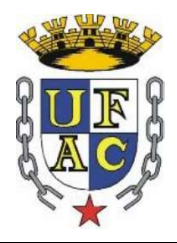 & $\begin{array}{c}\text { UÁQUIRI } \\
\text { Revista do Programa de Pós-Graduação em Geografia } \\
\text { UÁQUIRI - PPGGEO, v. 2, n. 1, p. } 128-143 \text {, ano } 2020 \\
\text { Home page: } \underline{\text { https://periodicos.ufac.br/index.php/Uaquiri }}\end{array}$ & PPG \\
\hline & & ISSN 2675-4088 \\
\hline
\end{tabular}

\title{
ESPACIALIZAÇÃO GEOGRÁFICA DA COVID-19 NA AMAZÔNIA SUL-OCIDENTAL: A CONTRIBUIÇÃO DA GEOGRAFIA DO RISCO NA GESTÃO DA PANDEMIA NO ESTADO DO ACRE - BRASIL
}

\author{
Anderson Azevedo Mesquita ${ }^{1,4^{*}}$, Rodrigo Otávio Peréa Serrano ${ }^{1,5}$, Fábio da Silva Lúcio ${ }^{2}$, \\ Jéssica Karine Barros Silva, Gabriel Brandão Xavier ${ }^{2}$, José Genivaldo do Vale Moreira ${ }^{3,5}$ \\ ORCID: http://orcid.org/0000-0003-0947-8070; https://orcid.org/0000-0002-7786-8305; \\ https://orcid.org/0000-0002-7273-7648; https://orcid.org/0000-0003-1194-3963; \\ https://orcid.org/0000-0001-8071-1862; https://orcid.org/0000-0002-2994-8482
}

${ }^{1}$ Professores da Universidade Federal do Acre, Centro de Filosofia e Ciências Humanas, Rio Branco, Acre, Brasil; ${ }^{2}$ Discente do Curso de Bacharelado em Geografia, Ufac, Universidade Federal do Acre, Rio Branco

Acre; Professor da Universidade Federal do Acre, Centro Multidisciplinar, Cruzeiro do Sul, Acre, Brasil; ${ }^{4}$ Doutorando do Programa de Pós-Graduação em Geografia da Universidade Federal de Rondônia; ${ }^{5}$ Professor do Programa de Pós-Graduação em Ciência e Tecnologia para a Amazônia, UFAC.

*amgeoufac@hotmail.com

Recebido em: 06/05/2020. Aceito em: 21/05/2020. Publicado em: 22/06/2020. DOI:

\section{RESUMO}

A sociedade contemporânea enfrenta seu maior desafio na a área de saúde. Trata-se de uma pandemia sem precedentes ocasionado por um vírus de origem zoonótica, que evoluiu ao ponto de infectar humanos, conhecido por novo coronavírus, causador da patologia denominada Covid-19. A doença já atingiu mais de 185 países, ocasionando mais de 200.000 mortes até a data 04/05/2020. No Brasil, impactou fortemente tanto o sistema de saúde quanto econômico. No Acre, os efeitos negativos estão em forte progressão. Nesse cenário, a geografia pode contribuir na compreensão da espacialização geográfica do fenômeno, especialmente no tocante à relação de risco existente, considerando as relações sociais e a tradução destas feições no espaço. Assim, neste trabalho objetiva-se compreender a dinâmica de disseminação e territorialização da Covid-19, no estado do Acre e, para isso, utilizou-se além dos preceitos da geografia do risco, as técnicas de geoprocessamento e da cartografia temática. A partir destes princípios foram elaborados mapas temáticos que representam a espacialização e a quantificação dos casos confirmados e de óbitos por Covid-19 ao longo dos meses de março e abril de 2020. Utilizouse como banco de dados as informações fornecidas diariamente através dos boletins epidemiológicos da Secretaria Estadual de Saúde. Por fim, concluiu-se que os casos confirmados de Covid-19 estão em forte expansão no estado, tendo como núcleo central de disseminação a capital Rio Branco e os municípios adjacentes. Os óbitos, durante o período de análise estão espacializados exclusivamente na capital, porém com destacada expansão nos últimos dias, o que indica existir um surto exponencial de crescimento da doença.

Palavras-chave: Pandemia; Covid-19; Geografia dos Riscos; Espacialização; Disseminação; Acre. 


\title{
GEOGRAPHICAL SPACIALIZATION OF COVID-19 IN THE SOUTH-WESTERN AMAZON: THE CONTRIBUTION OF RISK GEOGRAPHY IN THE MANAGEMENT OF PANDEMIA IN THE STATE OF ACRE
}

\begin{abstract}
Contemporary society faces its greatest challenge in the area of health. It is an unprecedented pandemic caused by a virus of zoonotic origin, which evolved to the point of infecting humans, known as a new coronavirus, which causes the pathology called Covid-19. The disease has reached more than 185 countries, causing more than 200,000 deaths to date 04/05/2020. In Brazil, it has strongly impacted both the health system and the economic system. In Acre, the negative effects are in strong progression. In this scenario, geography can contribute to the understanding of the geographical spatialization of the phenomenon, especially regarding the existing risk relationship, considering the social relations and the translation of these features in space. Thus, this work aims to understand the dynamics of dissemination and territorialization of Covid-19, in the state of Acre, and, for this, we used in addition to the precepts of risk geography, geoprocessing techniques and thematic cartography. Based on these principles, thematic maps representing the spatialization and quantification of confirmed cases and deaths by Covid-19 were elaborated during March and April 2020. The database used was the information provided daily through the epidemiological bulletins of the State Department of Health. Finally, it was concluded that the confirmed cases of Covid-19 are in strong expansion in the state, having a central nucleus of dissemination the capital Rio Branco and the adjacent municipalities. Deaths during the analysis period are spatialized exclusively in the capital, but with a prominent expansion in recent days, which indicates that there is an exponential outbreak of disease growth.
\end{abstract}

Keywords: Pandemic; Covid-19; Risk Geography; Spatialization; Dissemination; Acre.

\section{ESPACIALIZACION GEOGRAFICA DE COVID-19 EN EL AMAZONA SUROESTE: LA CONTRIBUCION DE LA GEOGRAPHY DE RIESGO EN LA GESTION PANDEMIC EN EL ESTADO DE ACRE}

\begin{abstract}
RESUMEN
La sociedad contemporánea se enfrenta a su mayor desafío en el ámbito de la salud. Es una pandemia sin precedentes causada por un virus de origen zoonótico, que evolucionó hasta el punto de infectar a los seres humanos, conocido como un nuevo coronavirus, que causa la patología llamada Covid-19. La enfermedad ha llegado a más de 185 países, causando más de 200.000 muertes hasta la fecha 04/05/2020. En Brasil, ha impactado fuertemente tanto el sistema de salud como el sistema económico. En Acre, los efectos negativos están en fuerte progresión. En este escenario, la geografía puede contribuir a la comprensión de la espacialización geográfica del fenómeno, especialmente en lo que respecta a la relación de riesgo existente, teniendo en cuenta las relaciones sociales y la traducción de estas características en el espacio. Así, este trabajo pretende entender la dinámica de difusión y territorialización de Covid-19, en el estado de Acre, y, para ello, utilizamos además de los preceptos de geografía de riesgo, técnicas de geoprocesamiento y cartografía temática. Sobre la base de estos principios, los mapas temáticos que representan la espacialización y cuantificación de los casos confirmados y las muertes por Covid-19 fueron elaborados durante los meses de marzo y abril de 2020. La base de datos utilizada es la información proporcionada diariamente a través de los boletines epidemiológicos del Departamento de Salud del Estado. Finalmente, se llegó a la conclusión de que los casos confirmados de Covid-19 se encuentran en fuerte expansión en el estado, teniendo como núcleo central de difusión la capital Río Branco y los municipios adyacentes. Las muertes durante el período de análisis se espacializan exclusivamente en la capital, pero con una expansión prominente en los últimos días, lo que indica que hay un brote exponencial de crecimiento de la enfermedad.
\end{abstract}

Palabras clave: Pandemia; Covid-19; Geografía de riesgo; Espacialización; Difusión Acre 


\section{INTRODUÇÃO}

Atualmente a humanidade enfrenta a maior crise global de saúde desde a propagação da gripe espanhola no início do século XX, que de acordo com Campos (2005), acometeu cerca de $30 \%$ da população mundial da época, dizimando mais de 40 milhões de vidas. Para Auerbach et al. (2013), os surtos de gripe sempre estiveram presentes no cotidiano das civilizações e assumiam diferentes feições de transmissibilidade e letalidade conforme contexto sociocultural e econômico que estavam inseridas.

Nesse cenário de evolução, em dezembro de 2019, quando os primeiros casos de uma doença pulmonar aguda foram registrados em Wuhan, província de Hubei, na China, e, após descartar-se várias outras síndromes já conhecidas tais como: influenza, influenza viária, (SARS-Co-V), e o (MERS), o mundo novamente entraria no que parecia ser um novo ciclo pandêmico relacionado a um surto de gripe.

Em janeiro de 2020, o governo chinês notificou a Organização Mundial da Saúde (OMS) informando que o patógeno causador do surto havia sido identificado com um novo coronavírus (SARS-Co-V2). Trata-se de um vírus de origem animal que conseguiu evoluir ao ponto de infectar humanos, provavelmente através do contato com animais silvestres utilizados para alimentação (NOVEL, 2020; LIU et al., 2020). Acredita-se que a primeira transmissão tenha ocorrido em um mercado ou feira ao ar livre nas imediações da cidade de Wuhan, e a partir desse foco inicial o vírus tenha se expandido rapidamente para todo o mundo (WU et al., 2020).

A Covid-19 é o nome dado a patologia que é ocasionada pelo novo vírus, este por sua vez pertence à família dos coronavírus e apresenta algumas similaridades com a SARS e a MERS. No entanto, o novo coronavírus possui características peculiares, que podem ser destacadas como a sua alta capacidade de disseminação espacial e bem sucedida estratégia de contágio, proporcionado o status de maior pandemia mundial do século em curso (HOLANDA, 2020).

O contágio por coronavírus pode ocorrer de pessoa para pessoa através de gotículas respiratórias, por contato direto com secreções infectadas e, recentemente, alguns estudos têm mostrado a possibilidade de transmissão por via aérea. Eventualmente, as gotículas podem ficar depositadas em diferentes superfícies, possibilitando o contato direto de indivíduos e que, ao levarem em contato com a boca, nariz ou olhos acabam por adquirindo a doença (AGUIAR et al., 2020). 
De acordo com dados compilados pela Johns Hopkins University e Medicine no Coronavirus Resource Center, até o dia 20 de abril de 2020 a Covid-19 já atingiu 185 países, com quase 2,5 milhões casos confirmados da doença e aproximadamente 170 mil óbitos atribuídos. Dentre os países mais afetados encontram-se EUA, Espanha e Itália que, respectivamente, confirmaram 783.290, 200.210 e 181.228 casos, até a data em destaque. Em relação ao total de mortes, os mesmos países se destacam: os EUA registraram 35.012, a Itália 24.114 e a Espanha 20.265 óbitos.

De acordo com Neto et al (2020), além da alta capacidade biológica do coronavírus, outro fator chave para a sua rápida disseminação se deu pela "intensa fluidez e capilaridade da circulação mundial". Em outras palavras, ou em termos biogeográficos, a elevada espacialização da Covid-19 se deu por "antropocaria", ou seja, o "homem moderno" e o seu estilo de vida globalizado permitiu, de forma instantânea, um maciço deslocamento intercontinental de pessoas, favorecendo a territorialização do vírus e a rápida disseminação pelo mundo.

No Brasil, o primeiro caso de covid-19 foi oficialmente registrado no dia 26 de fevereiro de 2020, no estado de São Paulo. Tratava-se de um homem que possuía histórico de viagem à região da Lombardia, na Itália, que naquele período tinha se tornado o epicentro de disseminação do coronavírus no mundo (CRODA et al., 2020). Dados do Ministério da Saúde indicam que no dia 20 de abril de 2020, o Brasil possuía 40.581 casos confirmados e um total de 2.575 óbitos por covid-19, saltando para 105.222 casos confirmados e 7.288 óbitos, quinze dias depois. No estado do Acre, os primeiros casos confirmados de covid-19 foram registrados no dia 17 de março de 2020, através de duas pessoas que tinham participado de um evento no estado de São Paulo. Até o dia 04 de maio de 2020, o estado confirmou 658 casos, com total de 25 óbitos por covid-19. Dos 22 municípios que compõe o estado 12 desses já registraram casos positivos da doença.

Para Beck (1992), a sociedade contemporânea pode ser traduzida como "a sociedade de risco", pois a distribuição do risco não considera às assimetrias sociais, econômicas ou geográficas. O risco tornou-se generalizado no espaço e no tempo, não obedecendo fronteiras físicas ou a um espaço estático e imutável. O risco é, portanto, dinâmico, flexível e caótico, e mesmo que a sua percepção possa ser abstrata ou materialmente improvável, sobretudo em função dos diferentes graus de vulnerabilidade, o fato é que os riscos compõem uma nova forma de ordem global, uma nova sociedade e uma nova forma de vida pessoal. 
É importante destacar que o conceito de risco é amplamente utilizado, pois trata-se de uma variável interdisciplinar que dialoga com os mais diversos campos do saber científico. Das ciências humanas às ciências naturais e exatas, o risco compõe um escopo de variações e interconexões com fenômenos materializados no espaço e, em função disso, com a geografia. Neste sentido, a geografia se apropria desta variável para compreender a intrínseca realidade construída a partir da relação do natural com o humano (ALMEIDA, 2011; GREGORY, 1992; WHITE et al, 2001).

De acordo com Marandola e Hogan (2004), o significado de risco (risk) é utilizado principalmente pelos geógrafos para indicar uma situação que poderá ocorrer no futuro e que, por conseguinte denota incerteza e insegurança. Os autores destacam que a origem do termo risco é complexa e pode assumir diversos significados, sendo alguns muito próximos e até idênticos aos conceitos de perigo ou hazards, por exemplo.

Para Leone e Vinet (2006), os geógrafos se destacam no estudo e compreensão de problemas sociais complexos por serem profissionais com formação pluridisciplinar e por terem visão de interface sobre fenômenos perigosos e os recursos vulneráveis, além da capacidade de administrar e manusear ferramentas cartográficas e de geoprocessamento. Logo, a Geografia do Risco seria a subárea da geografia responsável pela compreensão das diversas formas do risco, dentro os quais aqueles de origem e natureza multivariada como os naturais, os econômicos e os sociais (ALMEIDA, 2011).

Para Veyret (2007), a geografia do risco estuda a relação existente entre o risco considerando as relações sociais e a tradução destas feições no espaço. Por tal, infere-se que a geografia do risco surge como uma abordagem coerente para lidar com o problema da pandemia por covid-19. Ainda de acordo Veyret (2007, p. 12), "para o geógrafo, a tradução espacial do risco constitui um tema de estudo indispensável" e "as zonas de risco são definidas de formas e superfícies variáveis, mapeáveis em diferentes escalas espaciais e temporais".

A “tradução espacial do risco" mencionada pela autora só é possível através da análise sistêmica e integrada das diversas variáveis que compõe o risco. Dentre tais variáveis destacase, em princípio, a compreensão geoespacial do risco, ou seja, como um dado risco se comporta e se projeta no espaço geográfico? Qual a dimensão territorial do risco? Nestes termos, a ciência geográfica dispõe de ferramentas analíticas baseadas em técnicas cartográficas e de geoprocessamento para auxiliar no planejamento, resposta e mitigação dos riscos.

Neste sentido, considerando o quadro pandêmico enfrentando pelo mundo em função da covid-19, a geografia (especificamente a "geografia do risco", temática pesquisada pelo 
grupo de pesquisa Hidrologia, Meio Ambiente e Geografia dos Riscos) acaba por contribuir significativamente para compreensão regional do fenômeno. Assim, a geografia do risco pode auxiliar na identificação da lógica de espacialização e disseminação territorial da doença, avaliando a gestão, o planejamento, a mitigação e a reposta dos atores responsáveis pelo enfrentamento à pandemia, interpretando os possíveis impactos socioambientais e econômicos refletidos em uma região.

Em geral as técnicas cartográficas e de geoprocessamento realizam monitoramento remoto de vastas áreas do espaço geográfico, fornecendo uma gama de informações fundamentais sobre as variáveis do risco (BUFFON et al., 2018). De acordo com Bonfim et al. (2008), ao destacar a intrínseca relação entre a geografia e a epidemiologia, o uso de técnicas cartográficas e de geoprocessamento contribuem para a compreensão da organização do espaço, possibilitando maior aproximação dos fatores relacionados ao desenvolvimento das doenças e da sua distribuição entre os diversos grupos sociais.

Por fim, sob o olhar da geografia do risco, neste trabalho pretende-se colaborar para a compreensão da espacialização e disseminação territorial da covid-19 no estado do Acre a partir de técnicas de geoprocessamento e da cartografia temática. Trata-se de uma colaboração embrionária e descritiva sobre o fenômeno, mas que reflete um primeiro passo para entendê-lo de forma mais complexa e sistêmica no futuro. Este ensaio representa um dos primeiros de uma série de estudos que estão sendo desenvolvidos pelo grupo de pesquisa Hidrologia, Meio Ambiente e Geografia dos Riscos, da Universidade Federal do Acre.

\section{METODOLOGIA}

Conforme destacado por Bonfim et al. (2008), as técnicas de geoprocessamento e de cartografia temática surgem como importantes ferramentas para a compreensão espacial de diversos níveis do risco. Neste sentido, o presente ensaio faz uso de tais técnicas para compreender a evolução e disseminação da covid-19 no estado do Acre, considerando o primeiro caso relatado da doença no dia 17 de março de 2020, e a evolução dos cenários até o dia 04 de maio de 2020.

De acordo com o Instituto Brasileiro de Geografia e Estatísticas (IBGE), o estado do Acre (fig. 01) possui população estimada de 881.935 habitantes, densidade demográfica de 4,47 $\mathrm{hab} / \mathrm{km}^{2}$, renda per capita de $\mathrm{R} \$ 890,0,663$ de Índice de Desenvolvimento Humano (IDH), 0,557 de Índice de Gini, 73,8 \% de restrição a serviços de saneamento básico, 27,9\% de restrição 
à educação, 29,2\% de restrição a condições de moradia, 34,4\% dos habitantes possuem ensino fundamental incompleto. O Estado possui 22 municípios, cuja capital é a cidade de Rio Branco, que possui população estimada de 407.319 pessoas, concentrando $46,18 \%$ da população total do estado, além de possuir a maior densidade demográfica, estimada em $38,03 \mathrm{hab} / \mathrm{km}^{2}$ (IBGE, 2020).

Figura 1: Divisão geopolítica do Estado do Acre

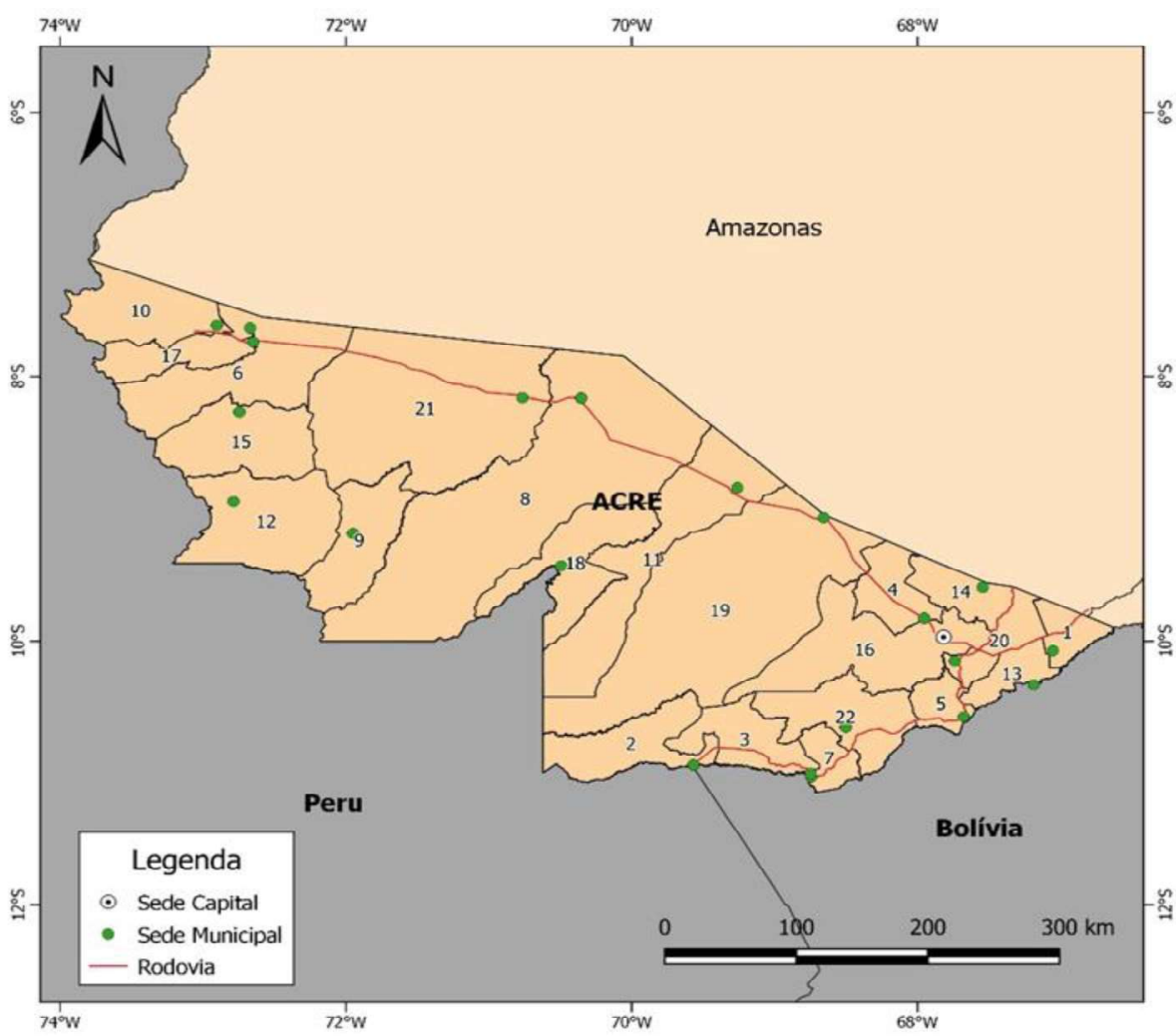

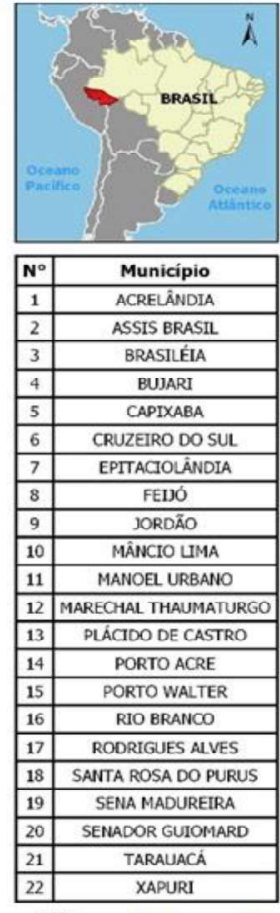

Ufac

Fonte: ZEE-AC, 2006 (Organizado pelo Laboratório do Grupo de Pesquisa Hidrologia, Meio Ambiente e Geografia dos Riscos, da Universidade Federal do Acre)

No presente ensaio, adotou-se como metodologia a elaboração de mapas, que foram projetados a partir de ferramentas de dois softwares: para tratamento, quantificação e organização dos dados brutos, utilizou-se o Microsoft Office Excel (2016), por meio do qual foram estruturadas as planilhas atualizadas diariamente, a partir de informações fornecidas pela Secretaria Estadual de Saúde do Acre. O banco de dados construído pelas planilhas facilitava a exportação das informações para serem plotadas nos mapas.

Para a construção dos mapas, adotou-se o QGIS (v. 3.4.12) LTR1, com licença de uso comum e gratuita. De acordo com Bruno (2017), os sistemas de informação geográfica (SIG) apresentam-se como ferramentas fundamentais para uma variedade de campos de pesquisa e tarefas científicas no campo das ciências ambientais, se aplicando perfeitamente à geografia do 
risco, mostrando-se como uma plataforma abrangente para processamento e disseminação de conhecimento.

Assim, em todo o processo de produção dos mapas, adotou-se as bases cartográficas contínuas, disponibilizadas pelo Instituto Brasileiro de Geografia e Estatística (IBGE) como a principal. Igualmente, foram utilizados os dados do Covid-19 (casos confirmados e óbitos), disponibilizados diariamente pela Secretaria de Estado da Saúde (SESACRE), por meio de boletins diários disponíveis no link: https://agencia.ac.gov.br/agencia-de-informacoes-sobrecoronavirus/.

A partir daí, foram criados dois projetos no referido software, cada um contendo uma variável a ser representada: casos e óbitos confirmados. Convém ressaltar, que foram criados mais dois projetos para ilustrar a evolução da dinâmica espacial da doença no estado do Acre, considerando intervalos de tempo de sete e quinze dias.

Em cada projeto, buscou-se padronizar um layout de uso comum, nas legendas e demais elementos, com a intenção de prover ao leitor informações transparentes e objetivas. Já na elaboração das legendas, foi adotado através da tabela de atributos, uma rotulação específica conforme os dados são inseridos, onde posteriormente no compositor de impressão foram salvos e exportados em arquivos do tipo (.jpeg e .pdf).

Por fim, é importante destacar que todos os mapas produzidos são disponibilizados diariamente em redes sociais, bem como estão disponíveis no site do Grupo de Pesquisa: Hidrologia, Meio Ambiente e Geografia Riscos, da Universidade Federal do Acre, com acesso gratuito através do link: https://onedrive.live.com/?authkey=\%21APKrpyeq5Bgr8w8\&id=4BA74D4C7FE8382F\%214 4005\&cid=4BA74D4C7FE8382F. No site, é possível acessar todos os mapas por temática (casos confirmados, notificações e óbitos), que estão apresentados em pastas conforme evolução mensal da covid-19 no estado.

\section{RESULTADOS E DISCUSSÃO}

\subsection{Da espacialização dos casos confirmados de Covid-19}

Conforme indicado na fig. 02 (a), o primeiro caso confirmado de covid-19 foi registrado no município de Rio Branco, no dia 17 de março de 2020. Até o dia 23 de março do ano corrente observa-se que o número total de casos evoluiu, contudo, de forma tímida. Portanto, na primeira semana epidemiológica, os casos de coronavírus concentraram-se na cidade de Rio Branco, 
tendo uma evolução de disseminação de 0 a 10 para a de 11 até 20 casos, a partir do dia 20 de março de 2020.

$\mathrm{Na}$ fig. 02 (b) observa-se a evolução da Covid-19 durante a segunda semana epidemiológica e, novamente, os casos se intensificaram na cidade de Rio Branco. O destaque se dá pela evolução da disseminação que no dia 24 de março ultrapassou o marco de 11 a 20 , para a de 21 até 30 casos de pessoas infectadas, e posteriormente ascendeu no dia 30 de março para a taxa de 31 até 40 casos confirmados.

Figura 2: (a, b): Espacialização de casos confirmados de Covid-19 (AC) - de 17 a 30/03/2020

a)

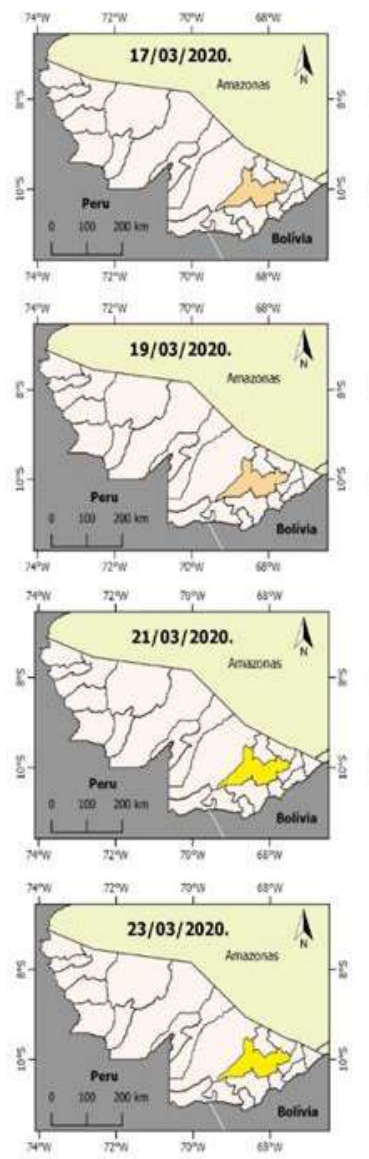

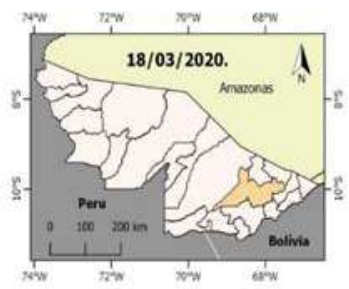
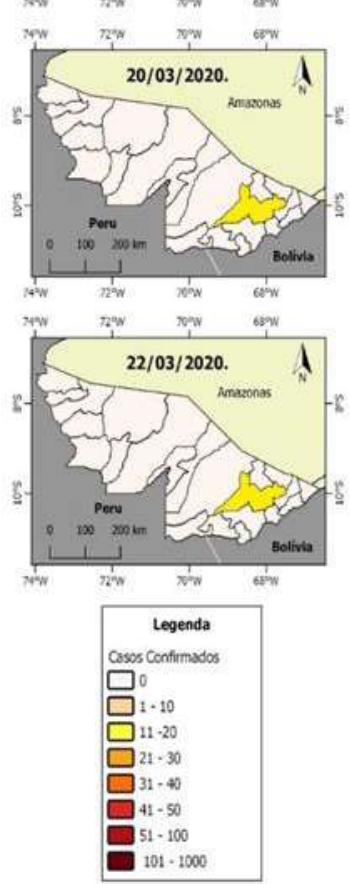

b)

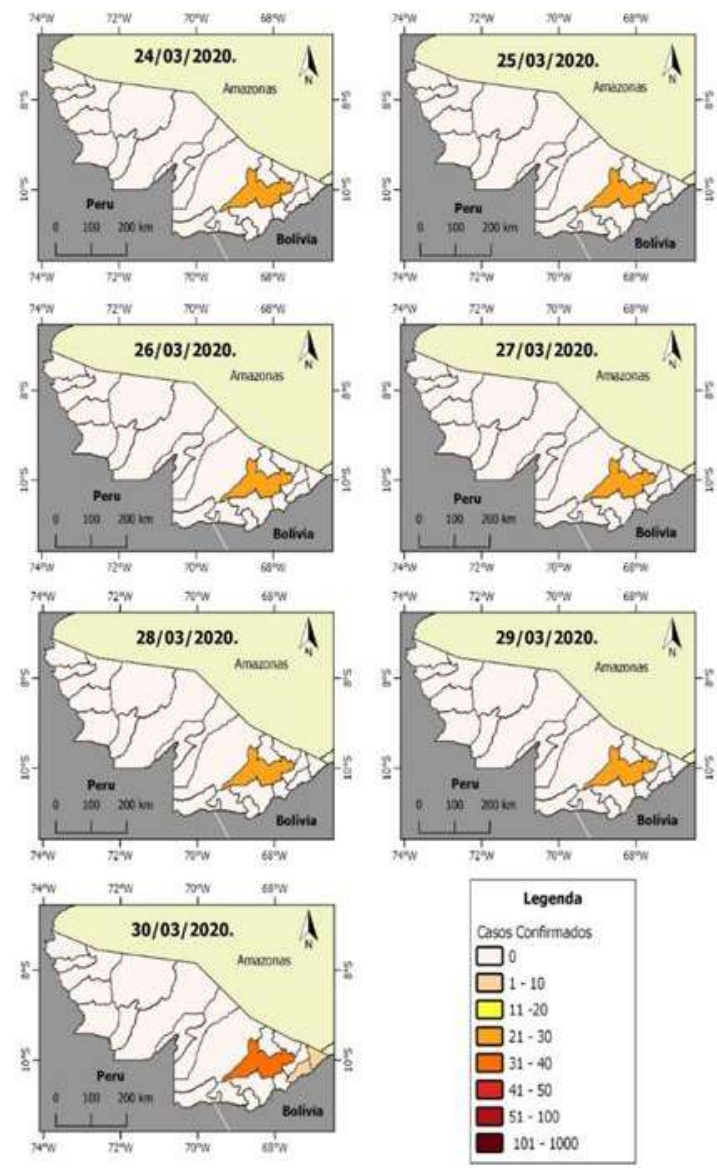

Outro fato relevante foi a confirmação dos primeiros casos da doença na cidade de Acrelândia e Porto Acre, que estão geograficamente próximas a Rio Branco. Tal proximidade fomenta a hipótese de motivação à extrapolação do vírus para além da capital do estado. Os primeiros casos de covid-19 registrados fora de Rio Banco ocorreram no dia 30 de março de 2020, e foram informados no $14^{\circ}$ boletim epidemiológico da Secretaria Estadual de Saúde. 
$\mathrm{Na}$ terceira semana epidemiológica os casos confirmados de covid-19 permaneceram na taxa de quantificação conforme demonstrado na fig. 03 (a). A novidade se dá pela espacialização da doença em mais um município, ou seja, além de Acrelândia e Porto Acre, a cidade de Plácido de Castro (06/04/2020) registrou seu primeiro caso de Covid-19. Observa-se que as cidades mantêm proximidade e destacada interação com a capital Rio Branco.

$\mathrm{Na}$ quarta semana epidemiológica observa-se que a disseminação do covid-19 se intensifica em Rio Branco e novamente a escala de quantificação de casos confirmados é extrapolada. Ou seja, de acordo com a fig. 03 (b), a partir do dia 08 de abril, Rio Branco inserese na escala de 41 até 50 casos confirmados. Também no dia 08 de março o primeiro caso é registrado no município de Bujari. Já os demais municípios mantem o total de casos dentro da escala quantitativa de 0 até 10 casos.

Figura 3: (a, b): Espacialização de casos confirmados de Covid-19 (AC) - de 31/03 até 13/04/2020

a)

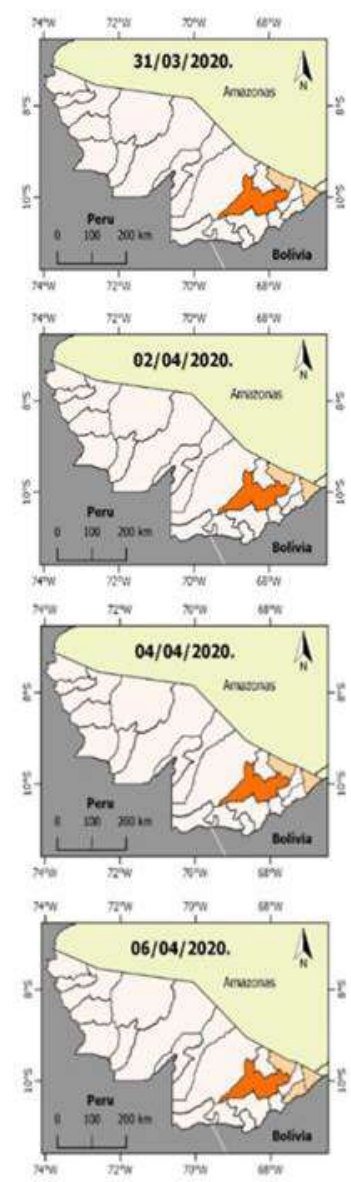

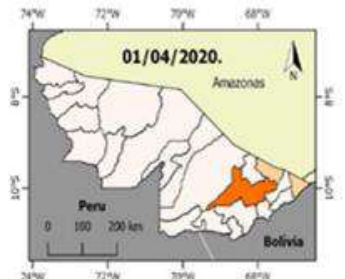
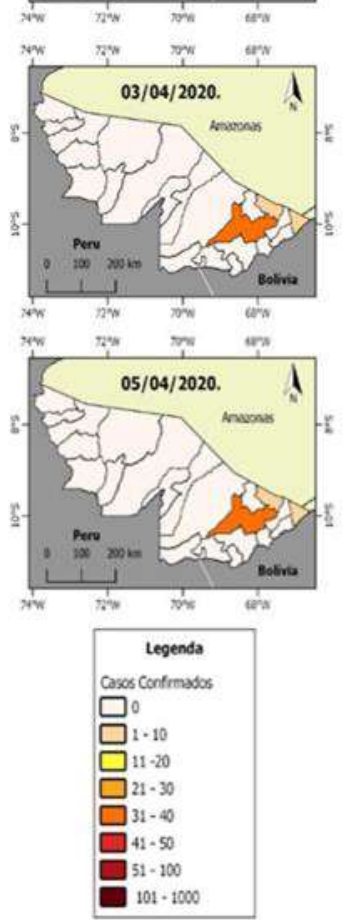

b)

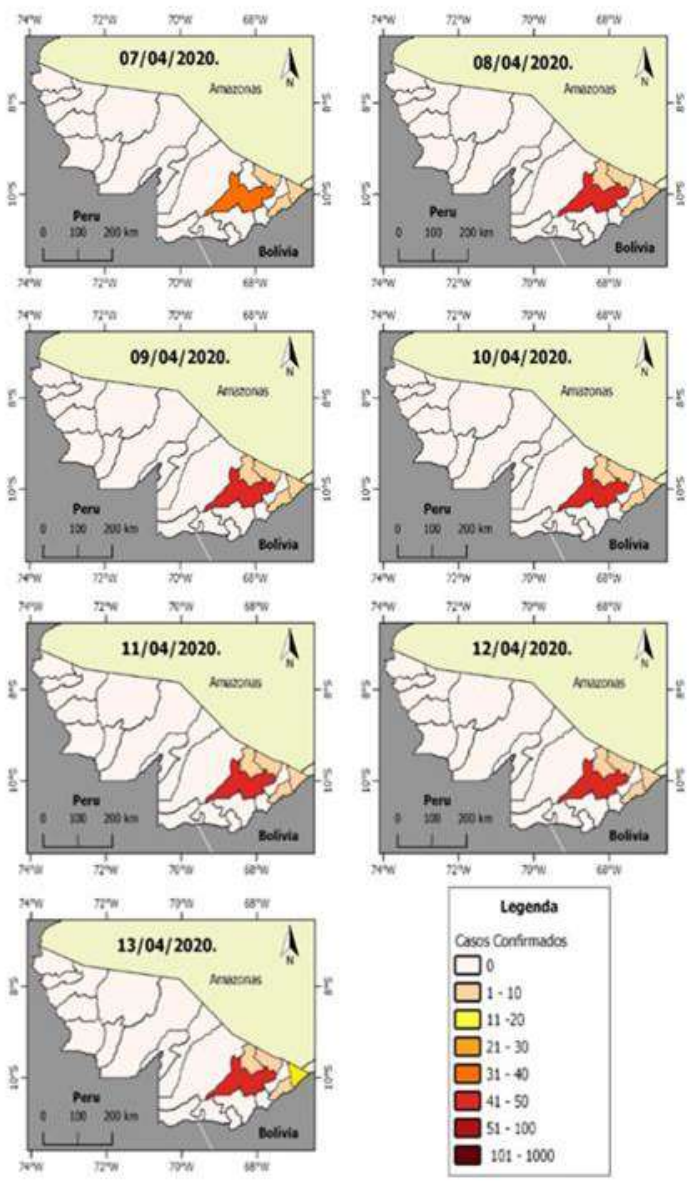

Os resultados da quinta semana epidemiológica indicam que a disseminação da covid19 se consolida nas cidades mais próximas à capital Rio Branco, ou seja, nas cidades que 
compõem a Regional do Baixo Acre. Neste tempo, as cidades de Cruzeiro do Sul (14/04/2020) e Senador Guiomard (19/04/2020) registram seus primeiros casos. De acordo com a fig. 04 (a), a escala de quantificação de Rio Branco se altera bruscamente de 41 até 50 para 101 até 1000 casos, cujo crescimento demonstra que a disseminação do vírus está atingindo escala exponencial.

Outro ponto relevante é o crescimento de casos nas cidades de Acrelândia e Plácido de Castro que, assim como a capital, sofreram extrapolação na escala quantitativa de casos, indicando progressão do total de infecções por covid-19. Cruzeiro do Sul, a segunda maior cidade do estado em quantitativo populacional, registra seus primeiros casos o que pode traduzir que futuramente o estado tenha um segundo núcleo de expansão da doença, agora concentrada na região do Juruá. Na fig. 04 (b) observa-se que na última semana epidemiológica, os casos de Covid-19 se intensificaram na cidade de Plácido de Castro, que atingiu o patamar de 31 até 40 casos confirmados.

Figura 4: (a, b): Espacialização de casos confirmados de Covid-19 (AC) - de 14 a 27/04/2020

a)

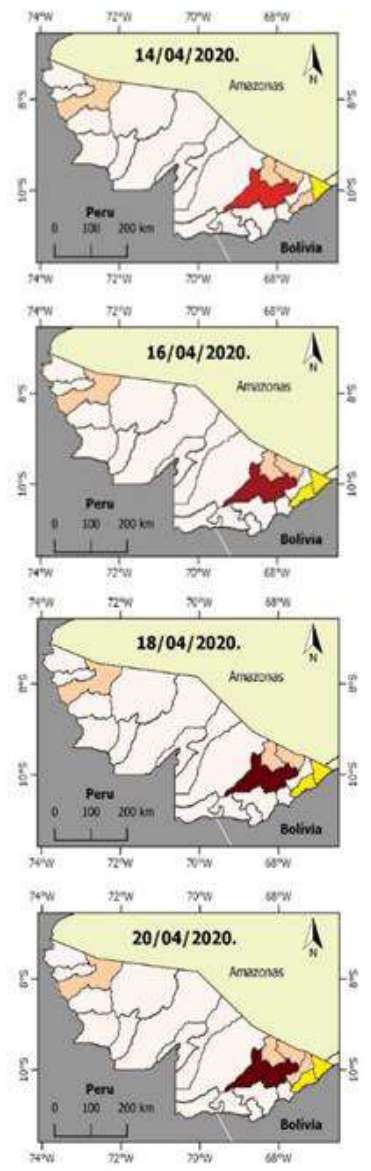

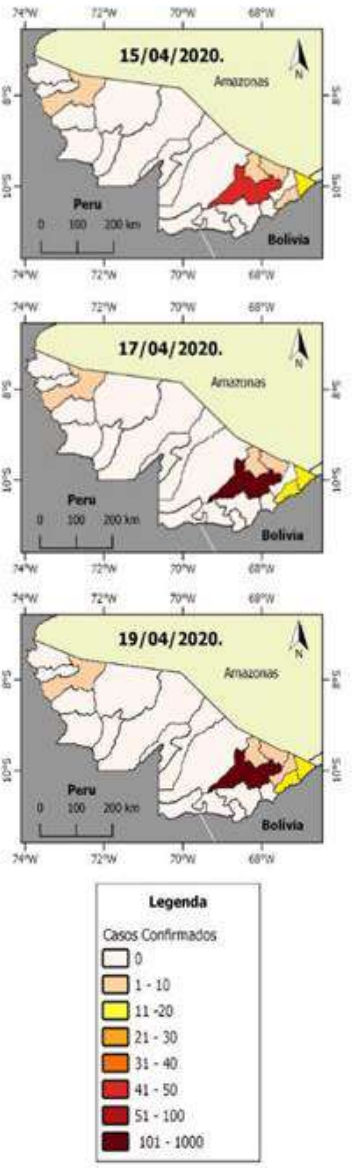

b)
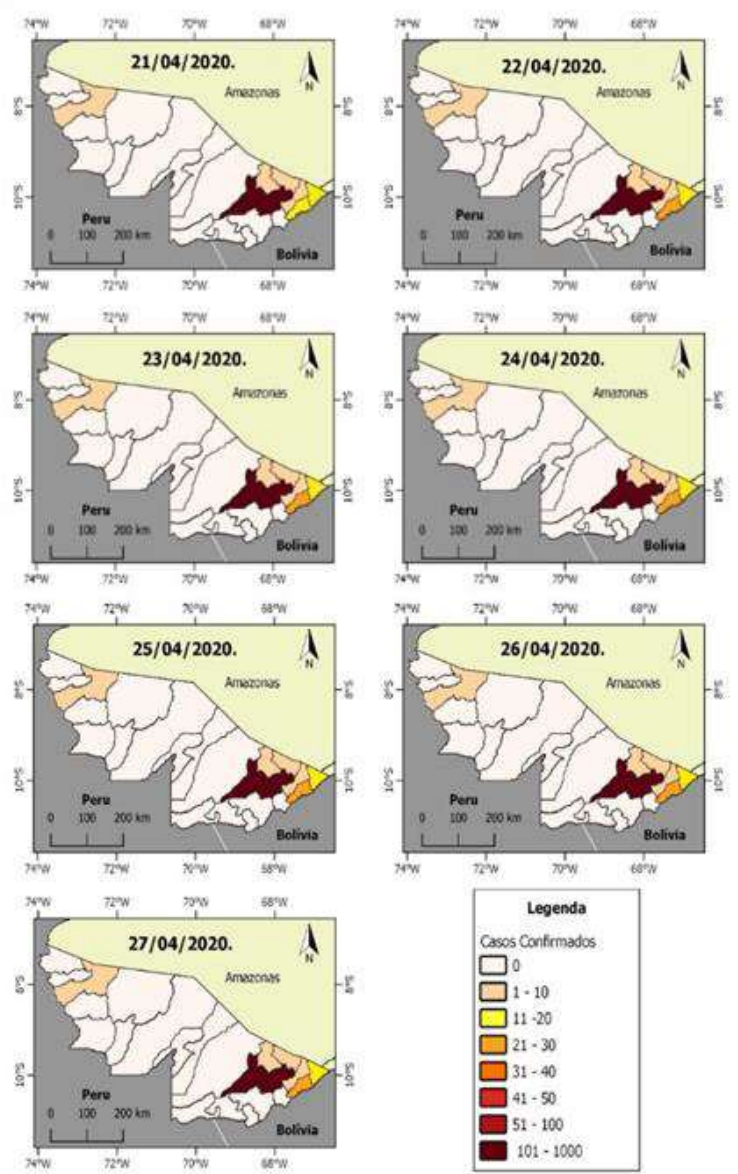


\section{a. Da espacialização dos casos de óbitos de Covid-19}

Para analisar a espacialização dos óbitos por covid-19 no estado, é preciso destacar, a priori, que o período de análise se concentra entres os dias 06/03 e 04/05/2020. Outra vertente importante é compreender como se dá a evolução da doença, ou seja, desde a transmissão até o comprometimento crítico que leva alguns pacientes a necessitarem de ventilação mecânica ou acesso à Unidade de Tratamento Intensiva (UTI).

Todo o processo de evolução da doença e o seu potencial de letalidade pode variar por uma série de fatores, que vão desde o acesso e a qualidade da estrutura médica hospitalar disponível para atendimento, e principalmente o histórico de comorbidades do paciente. Assim, tendo como critérios tais variáveis, é possível inferir que o tempo médio para a evolução de uma fatalidade por covid-19 pode superar um período de 10 até 20 dias. Este período, por lógica, acaba por influenciar no acompanhamento e na estimação da evolução do número de óbitos no estado.

A fig. 5 demonstra que os primeiros óbitos por covid-19 foram registrados no dia 06 de abril de 2020, sendo um na capital Rio Branco e outro no município de Plácido de Castro. Destaca-se que o primeiro óbito ocorreu 21 dias depois do primeiro caso registrado no estado, que ocorreu no dia 17 de março. De acordo com o gráfico observa-se que até o dia 23/04 os casos acumulados de óbitos seguem um "comportamento linear" e gradual, no entanto, a partir desta data a tendência de crescimento acelera, indicando "comportamento exponencial" e abrupto que, obviamente, precisa de comprovação matemática.

Considerando a espacialização dos óbitos, a fig. 6 indica concentração na cidade de Rio Branco, com viés de crescimento exponencial para os próximos dias, como se indica na figura 5. Além da capital, Acrelândia e Plácido de Castro foram as cidades que registraram óbitos, seguindo a tendência observada na territorialização do número de casos confirmados de Covid19.

No entanto, estima-se que conforme a doença avance para o interior do estado, considerando-se um cenário de possível colapso do sistema de saúde, os óbitos também acabem por fazer parte de uma triste estatística desses municípios. 
Figura 5: Distribuição do ${ }^{\circ}$ de óbitos acumulados da Covid-19, por data do óbito, AC, 2020

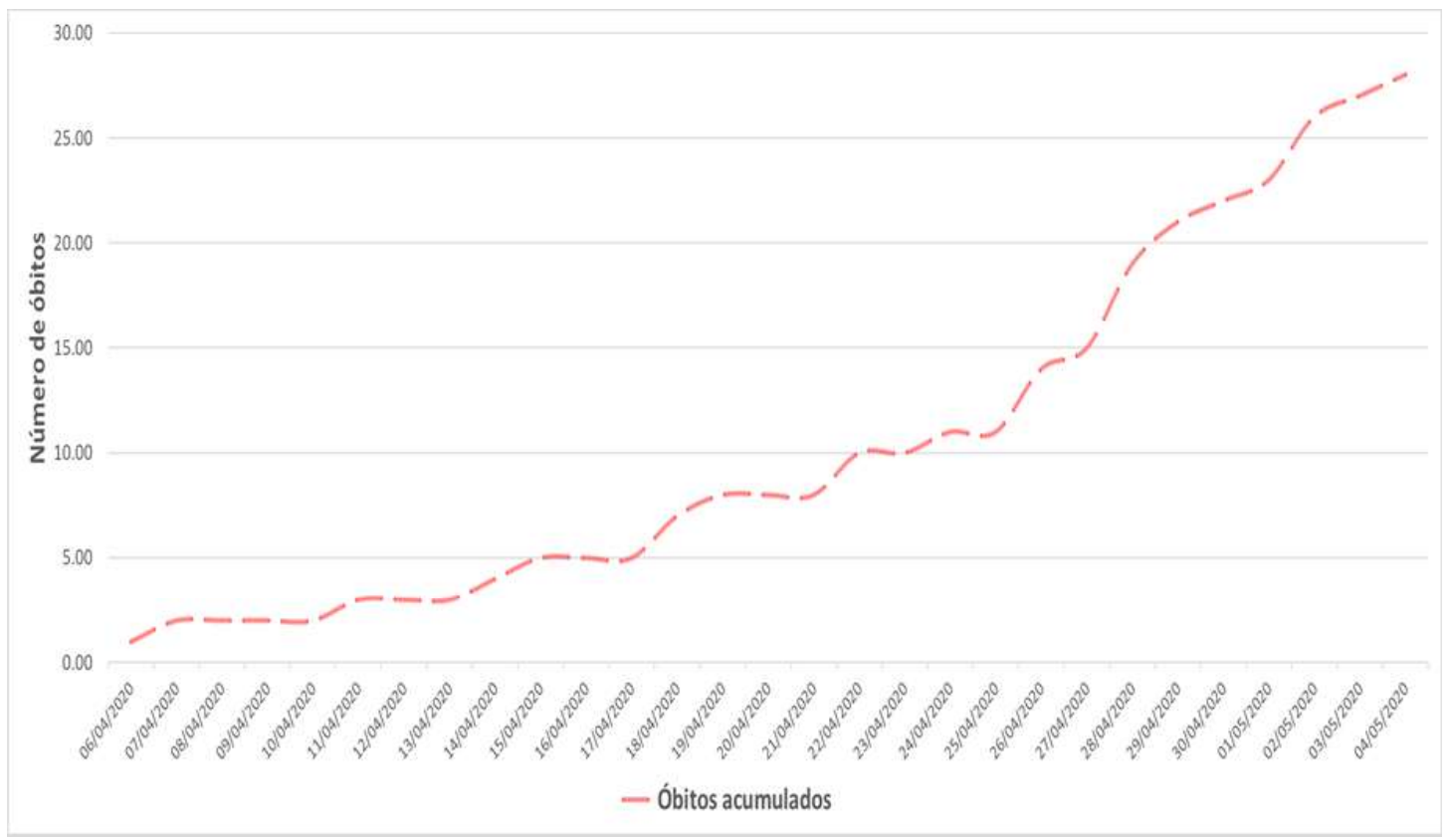

Fonte: Secretaria de Estado de Saúde - AC (2020) adaptado pelos autores.

Figura 6: Distribuição acumulada de óbitos no estado do Acre - 06/04 a 04/05/2020
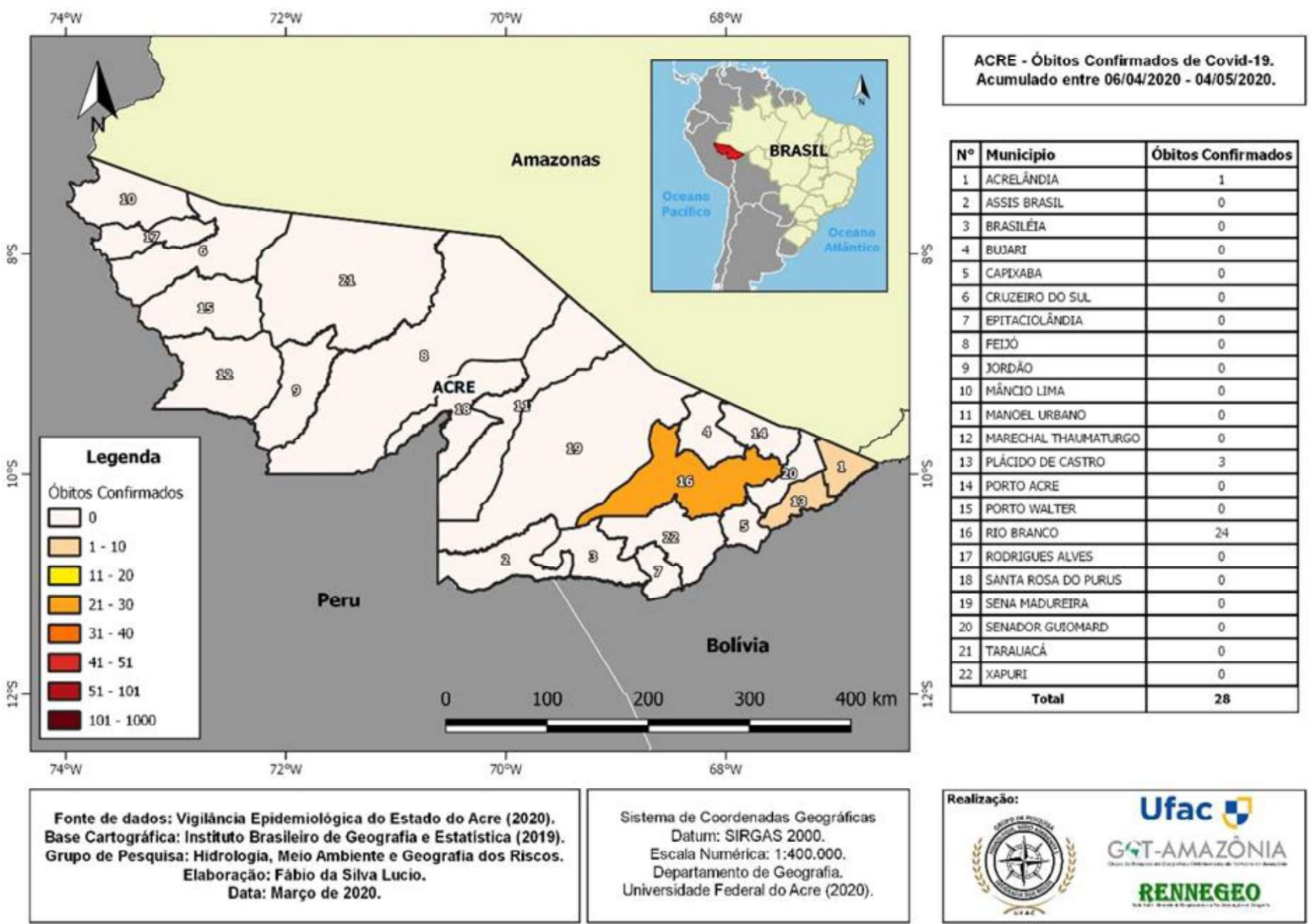


\section{CONSIDERAÇÕES FINAIS}

O presente estudo se propôs a analisar a disseminação da Covid-19 no estado do Acre, no âmbito espacial e temporal, tendo como referência os conceitos basais da geografia do risco. Os resultados permitiram convergir para os seguintes cenários a partir do total de casos confirmados bem como do número total de óbitos:

- Há uma concentração dos casos confirmados na capital Rio Branco com tendência de crescimento exponencial;

- A exceção de Cruzeiro do Sul, o maior volume de casos confirmados estão espacializados em municípios adjacentes a capital Rio Branco, o que indica ser a região o grande epicentro de contaminação do estado;

- Observou-se que a disseminação dos casos ocorreu primeiramente ao longo da BR 364 e entres os municípios com maior fluxo de pessoas com a capital;

- Em relação ao total de óbitos, observa-se que durante o período analisado especializaram-se quase totalmente na capital Rio Branco, o que já era esperado uma vez que a cidade também concentra o maior número de casos;

- O número de óbitos vem acelerando nos últimos dias que compreenderam a análise deste trabalho, o que pode indicar, assim como no número de casos confirmados, que o estado passará por uma situação de crescimento exponencial desta variável.

Por fim, ressalta-se que este estudo é apenas preliminar e não engloba, portanto, a maior parte do desenrolar da pandemia. No entanto, a metodologia proposta demonstra que a geografia, e em especial a geografia do risco pode colaborar efetivamente para o conhecimento da disseminação da pandemia na região.

\section{REFERENCIAS:}

ALMEIDA, Lutiane Queiroz de. Por uma ciência dos riscos e vulnerabilidades na geografia (a science of the risks and vulnerabilities to geography). Mercator, Fortaleza, v. 10, n. 23, p. 83 a 99, nov. 2011. ISSN 1984-2201. DOI: https://doi.org/10.4215/RM2011.1023.0007

AGUIAR, Ana. Da emergência de um novo vírus humano à disseminação global de uma nova doença-Doença por Coronavírus 2019 (COVID-19). Instituto de Saúde Pública da Universidade do Porto, 2020. Disponível em: http://asset.youoncdn.com/ab296ab30c207ac641882479782c6c34/7c7b39d50c8250c4b32f99 1c3245e5f7.pdf $>$ Acesso em: 15 maio 2020. 
AUERBACH, P.; OSELAME, G. B.; DE ALMEIDA D. D. Revisão Histórica da Gripe no mundo e a nova H7N9. Revista de Medicina e Saúde de Brasília, n2, v3, 2014.

BARCELlOS, C. Elos entre geografia e epidemiologia. Cad. Saúde Pública, Rio de Janeiro, v. 16, n. 3, p. 607-609, Sept. 2000. Available from http://www.scielo.br/scielo.php?script $=$ sci arttext\&pid=S0102-

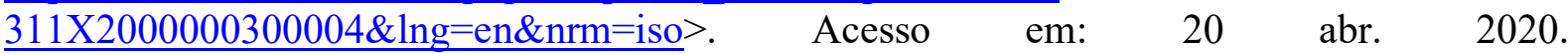

DOI: https://doi.org/10.1590/S0102-311X2000000300004.

BECK, U. Risk Society. Towards a new modernity. Londres: Sage Publications, 1992.

BONFIM, C.; MEDEIROS, Z. Epidemiologia e geografia: dos primórdios ao geoprocessamento. Espac Saude, v.10, n.1, p.53-62, 2008

BRUNO, L. O. Aplicabilidade de Sistemas de Informações Geográficas (SIGs) livres nas ciências ambientais: o uso do QGIS. Revista Brasileira de Gestão Ambiental e Sustentabilidade, v. 4, n. 8, p. 321-326, 2017.

BUFFON, E. A. M.; DA PAZ, O. L. de S. Geoprocessamento para mapeamento das áreas de risco de inundações: uma aplicação na sub-bacia hidrográfica do Rio Palmital, Colombo e Pinhais-Paraná (Geoprocessing for mapping flood risk areas: an application in the Palmital river watershed (Colombo and Pinhais-Paraná)). Revista Brasileira de Geografia Física, v. 11, n. 6, p. 2186-2200, 2018.

Campos HS. Influenza, uma nova tsunami. Pulmão RJ, v. 14, n. 2, p. 104-108, 2005

Center for Systems Science and Engineering at Johns Hopkins University \& Medicine. Disponível em: https://coronavirus.jhu.edu/map.html. Acesso em: 14 abr. 2020.

CRODA, J. OLIVEIRA, W. K. de; FRUTUOSO, R. L.; MANDETTA, L. H.; BAIA-DASILVA, D. C.; BRITO-SOUSA, J. D.; MONTEIRO, W. M.; GUIMARÃES, M. V. COVID-19 in Brazil: advantages of a socialized unified health system and preparation to contain cases. Rev. Soc. Bras. Med. Trop. Uberaba, v. 53, e20200167, 2020. Available from < http://www.scielo.br/scielo.php?script=sci arttext\&pid=S0037-

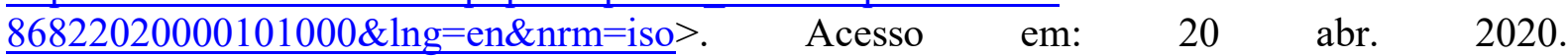
DOI: https://doi.org/10.1590/0037-8682-0167-2020.

GREGORY, Kennett. J. A natureza da Geografia Física. (trad. Eduardo de A. Navarro). Rio de Janeiro: Bertrand Brasil, 367p., 1992.

HOLANDA, Vanderlan Nogueira. Pandemia de Covid-19 e os esforços da ciência para combater o novo coronavírus. Revista Interfaces: Saúde, Humanas e Tecnologia, v. 8, n. 1, p. 360-361, 2020.

BRASIL. IBGE. IBGE estados: panoramas, 2020. Disponível em: https://cidades.ibge.gov.br/brasil/ac/panorama

LEONE, F..; VINET, F. La vulnerabilité, un concept fondamental au coeur des méthodes d'évaluation des risques naturels. In : LEONE, F.; VINET, F. La vulnerabilité des sociétés et 
des territoires face aux menaces naturelles: analyses géographiques. Montpellier : Université Paul Valery, Collection Géorisques n. 1, 144p., 2006

LIU, Y.; GAYLE, A. A.; WIDER-SMITH, A.; ROCKLOV, J. The reproductive number of COVID-19 is higher compared to the SARS coronavirus. Travel Medicine Journal, v. 27, ed. 2, 2020. DOI: https://doi.org/10.1093/jtm/taaa021

MARANDOLA JR, E.; HOGAN, D. J. Natural hazards: o estudo geográfico dos riscos e perigos. Ambiente\&Sociedade, v. 7., n. 02. 2004.

NETO, T. O.; GARCIA, T. de S. L.; SPINUSSI, E. Pandemia de COVID-19, as fronteiras pelo mundo e o transporte aéreo na Itália. Confins. Revue franco-brésilienne de géographie/Revista franco-brasilera de geografia, v. 44, 2020. DOI: $\underline{\text { https://doi.org/10.4000/confins. } 27577}$

NOVEL, Coronavirus Emergency Response Epidemiology Pneumonia, et al. The epidemiological characteristics of an outbreak of 2019 new coronavirus diseases (COVID19) in China. Zhonghua liu xing bue xue za zhi = Zhonghua liuxingbingxue zazhi, v. 41, n. 2, 145p., 2020 DOI: https://doi.org/10.3760/cma.j.issn.0254-6450.2020.02.003

VEYRET, Y. Os riscos: o homem como agressor e vítima do meio ambiente. In: Os riscos: o homem como agressor e vítima do meio ambiente. 2007.

WHITE, G. F.; KATES, R. W.; BURTON, Ian. Knowing better and losing even more: the use of knowledge in hazards management. Environmental hazards. v. 3. 2001.

WU, Z.; MCGOOGAN, J. M. Features and important lessons from the 2019 coronavirus disease outbreak (COVID-19) in China: summary of a report of 72314 cases from the Chinese Center for Disease Control and Prevention. Jama, 2020. DOI: https://doi.org/10.1001/jama.2020.2648 